\title{
Significant association between 53 BP1 expression and grade of intraepithelial neoplasia of esophagus: Alteration during esophageal carcinogenesis
}

\author{
Nozomi Ueki $^{\mathrm{a}}$, Yuko Akazawa ${ }^{\mathrm{a}, \mathrm{b}, 1}$, Shiro Miura ${ }^{\mathrm{a}, 1}$, Katsuya Matsuda ${ }^{\mathrm{a}}$, Hirokazu Kurohama ${ }^{\mathrm{a}}$, \\ Toshinobu Imaizumi ${ }^{a}$, Hisayoshi Kondo ${ }^{c}$, Masahiro Nakashima ${ }^{a}$,* \\ ${ }^{a}$ Department of Tumor and Diagnostic Pathology, Atomic Bomb Disease Institute, Nagasaki University Graduate School of Biomedical Sciences, 1-12-4 Sakamoto, Nagasaki \\ 852-8523, Japan \\ ${ }^{\mathrm{b}}$ Department of Gastroenterology and Hepatology, Nagasaki University Hospital, 1-7-1 Sakamoto, Nagasaki 852-8501, Japan \\ ${ }^{\mathrm{c}}$ Biostatistics Section, Division of Scientific Data Registry, Atomic Bomb Disease Institute, Nagasaki University, 1-12-4 Sakamoto, Nagasaki 852-8523, Japan
}

\section{A R T I C L E I N F O}

\section{Keywords:}

P53-binding protein 1

DNA damage response

Genomic instability

Esophageal carcinoma

Squamous cell carcinoma

\begin{abstract}
A B S T R A C T
Background: Abnormal DNA damage response (DDR) leads to genomic instability and carcinogenesis. P53binding protein 1 (53 BP1), a DDR molecule, is known to accumulate at the sites of DNA double-strand breaks. The aim of this study was to analyze the expression pattern of 53 BP1-nuclear foci (NF) in esophageal neoplasms in order to visualize the state of DDR in esophageal carcinogenesis and to clarify its significance in the molecular pathology of the disease.

Methods: A total of 61 lesions from 22 surgically resected samples of esophageal cancer, including histologically normal squamous epithelium, low-grade intraepithelial neoplasia (LG-IN), high-grade intraepithelial neoplasia (HG-IN), carcinoma in situ (CIS), and invasive squamous cell carcinoma (SCC), were included in the study. 53 BP1 and Ki-67 expression were analyzed by double-labeled immunofluorescence.

Results: The number of discrete $53 \mathrm{BP} 1-\mathrm{NF}$ increased as the tumor progressed from normal epithelium through LG-IN, HG-IN, CIS, and SCC. 53 BP1-NF larger than $1 \mu \mathrm{m}$ in diameter (large foci), indicating intensive DDR, also showed a stepwise increase during the progression of carcinogenesis. Of note, large foci of 53 BP1 were found in significantly higher numbers in HG-IN than in LG-IN. Furthermore, localization of 53 BP1-NF in Ki-67-positive cells, indicating the abnormal timing of DDR, also increased with malignancy progression.

Conclusions: 53 BP1-NF accumulation increases during cancer progression from LG-IN to HG-IN to CIS to SCC. Detection of 53 BP1-NF by immunofluorescence, especially large foci, is a feasible method of estimating DNA instability and the malignant potential of esophageal intraepithelial neoplasia.
\end{abstract}

\section{Introduction}

Esophageal cancer is the sixth most common cause of cancer-related deaths worldwide [1]. There are two major subtypes of esophageal cancer: squamous cell carcinoma (SCC) and adenocarcinoma. While adenocarcinomas are prevalent in western countries, SCC is more common in Asian countries, such as Japan. The development of esophageal SCC is considered a multistep process that progresses from normal squamous epithelium to intraepithelial neoplasia to carcinoma [2], a process that has been genetically confirmed by whole-genome sequencing (WGS) [3]. Intraepithelial neoplasia in the esophagus is further classified into low-grade intraepithelial neoplasia (LG-IN) and high-grade intraepithelial neoplasia (HG-IN); LG-IN refers to when the neoplasia occupies less than half of the epithelium, whereas HG-IN

\footnotetext{
Abbreviations: NF, nuclear foci; LG-IN, low-grade intraepithelial neoplasia; HG-IN, high-grade intraepithelial neoplasia; CIS, carcinoma in situ; SCC, squamous cell

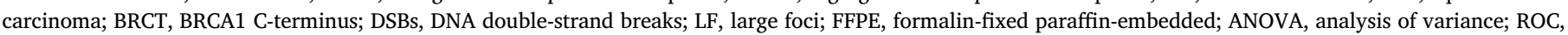
receiver operating characteristic; WGS, whole-genome sequencing; DDR, DNA damage response; GIN, genomic instability; 53 BP1, P53-binding protein 1

* Corresponding author.

E-mail addresses: nozominakamuraster@gmail.com (N. Ueki), akazaway@nagasaki-u.ac.jp (Y. Akazawa), miura610@nagasaki-u.ac.jp (S. Miura), katsuya@nagasaki-u.ac.jp (K. Matsuda), hiro8361@gmail.com (H. Kurohama), toshinobu0608@nagasaki-u.ac.jp (T. Imaizumi), hkondo@nagasaki-u.ac.jp (H. Kondo), moemoe@nagasaki-u.ac.jp (M. Nakashima).

${ }^{1}$ These authors contributed to equally to this work.
} 
refers to lesions involving more than half of the epithelium (WHO classification, 2010) [2]. When the full thickness of the epithelium is involved, it is classified as carcinoma in situ (CIS). HG-IN, CIS, and early SCC limited to the lamina propria are suitable for endoscopic treatment [4,5]. As the prognosis of advanced esophageal SCC is poor, it is of clinical interest to detect lesions suitable for treatment during the early phase. Identifying the extent of DNA damage response (DDR) occurring during SCC development may provide beneficial implications for early diagnosis and identifying appropriate therapeutic strategies.

Genomic instability (GIN) is thought to play an essential role in cellular transformation during SCC pathogenesis [3]. Tumor protein53-binding protein $1(53 \mathrm{BP} 1)$ is a large $(350 \mathrm{kD})$ multi-domain protein that belongs to a family of evolutionarily conserved DDR proteins with BRCA1 C-terminus (BRCT) domains [6-9]. 53 BP1 is a nuclear protein that localizes rapidly to sites of DNA double-strand breaks (DSBs) and activates p53, along with other kinases [8,10-14]. Activated kinases play a critical role in DDR, including, the induction of cell cycle arrest, DNA repair, and apoptosis $[15,16] .53 \mathrm{BP} 1$ has also been well described as a marker for DDR. It has been well documented (viaimmunofluorescence) that 53 BP1 exhibits diffuse nuclear expression in untreated primary cells and is localized at DSB sites, forming discrete nuclear foci (NF) after radiation exposure in vitro $[8,10,17,18]$. In addition to activation by exogenous stimuli, $53 \mathrm{BP} 1$ also forms NF during endogenous DDR during malignant transformation. Since GIN is triggered by intensive endogenous DDR [19,20], 53 BP1 expression can serve as a potential marker for estimating the level of GIN. We previously demonstrated a correlation between the appearance of 53 BP1 and the malignant potential of human tumors, including thyroid [21], skin [22], and uterine cervical cancers [23]. It appears that the presence of $53 \mathrm{BP} 1-\mathrm{NF}$ larger than $1.0 \mu \mathrm{m}$ in diameter (large foci: LF) represents prolonged DDR and is closely associated with a higher level of cervical carcinogenesis [23]. In the current study, we attempted to clarify the utility of 53 BP1 expression as a molecular marker of DNA instability in esophageal squamous neoplasms.

\section{Material and methods}

\subsection{Patients and histological evaluation}

This study was conducted retrospectively in accordance with the Declaration of Helsinki and was approved by the Institutional Ethical Committees for Medical Research at Nagasaki University (approval date: July 24, 2015; \#15,062,617).

A total of 61 lesions from 22 surgically resected, formalin-fixed, paraffin-embedded (FFPE) esophageal cancer tissues were selected for the study; these included (1) normal squamous epithelium without nuclear or cellular atypia outside the margin of the tumor (12 sites), (2) LG-IN (9 sites), (3) HG-IN (9 sites), (4) CIS (12 sites), (5) surface lesions of SCC (within $1000 \mu \mathrm{m}$ of the tumor surface, 9 sites), and (6) deep lesions of SCC (invasive front of the tumor, 10 sites; Table 1). Two pathologists independently confirmed the diagnosis. When there was a difference in opinion, the final diagnosis was made after discussing and

Table 1

Summary of subjects used in this study.

\begin{tabular}{lllll}
\hline Histological type & $\mathrm{n}$ & $\begin{array}{l}\text { Sex [M/ } \\
\text { F] }\end{array}$ & $\begin{array}{l}\text { Mean age [years } \\
\text { (range) }\end{array}$ & $\begin{array}{l}\text { p53 positive rate } \\
(\%)\end{array}$ \\
\hline Normal & 12 & $10 / 2$ & $71(54-79)$ & 0 \\
LG-IN & 9 & $9 / 0$ & $70.5(63-76)$ & 11.1 \\
HG-IN & 9 & $8 / 1$ & $71(54-78)$ & 55.6 \\
CIS & 12 & $12 / 0$ & $69(54-79)$ & 58.3 \\
SCC(surface) & 9 & $9 / 0$ & $69(62-79)$ & 33.3 \\
SCC(deep) & 10 & $10 / 0$ & $70(54-79)$ & 20.0 \\
\hline
\end{tabular}

LG-IN: low-grade intraepithelial neoplasia, HG-IN: High-grade intraepithelial neoplasia, CIS: carcinoma in situ, SCC: squamous cell carcinoma. considering the structural atypia (cell density, cell differentiation, and cell polarity) and cell atypia (nucleus size, nuclear irregularity, amount of nuclear chromatin, polarity, clarity of nucleolus, nucleus/cytoplasm ratio, and abnormal mitosis). Representative images of the normal esophageal epithelium, LG-IN, HG-IN, CIS, and invasive SCC are shown in Supplementary Fig. 1. P53 expression was assessed by immunohistochemistry to assure histological diagnosis.

\subsection{Immunohistochemistry}

\subsubsection{Immunofluorescence analysis of $53 \mathrm{BP} 1$}

53 BP1-NF formation and expression of Ki-67 were assayed using double-labeled immunofluorescence to assess the extent of DDR. After antigen retrieval by exposure to microwaves in citrate buffer, deparaffinized sections were pre-incubated with $10 \%$ normal goat serum. Tissues were then incubated with a mixture of rabbit anti-53 BP1 (1:200; Catalog No.A300-272A, Bethyl Laboratories, Montgomery, TX, USA) and monoclonal mouse anti-Ki-67 (MIB-1; 1:50; Catalog No.M7240, Dako, Jena, Deutschland) antibodies, followed by incubation with a mixture of Alexa Fluor 488-conjugated goat anti-rabbit and Alexa Fluor 546-conjugated goat anti-mouse antibodies (Invitrogen, Carlsbad, CA, USA). Specimens were counterstained with DAPI-I (Vysis, Downers Grove, IL, USA) and then analyzed and imaged using a high standard all-in-one fluorescence microscope (Biorevo BZ-9000; Keyence, Osaka, Japan). Five fields were observed for each sample at a magnification of $1000 \times$. Samples were classified into three groups based on 53 BP1 expression, as described in previous studies (Fig. 2) [21,23-25]. Stable or weak staining of 53 BP1 in the nucleus was defined as a stable type, discrete NF as unstable type, and discrete NF larger than $1.0 \mu \mathrm{M}$ in diameter as LF type. Next, we calculated the percentage of epithelial nuclei with different levels of 53 BP1 expression. In selected samples, the percentage of 53 BP1-NF-containing nuclei in Ki-67-positive cells was also calculated.

\subsubsection{Immunohistochemistry of $p 53$}

For the assessment of p53 expression, 4- $\mu \mathrm{m}$-thick sections were cut and mounted on a silane-coated glass slide, deparaffinized, and soaked for $15 \mathrm{~min}$ at room temperature in $0.3 \% \mathrm{H}_{2} \mathrm{O}_{2}$ /methanol to block endogenous peroxidases. Immunostaining was performed with mouse anti-p53 monoclonal antibody (DO7; 1:50; Catalog No.M7001, Dako) at $4{ }^{\circ} \mathrm{C}$ overnight. The primary antibody was visualized using the Histofine Simple Stain MAX-PO(M) Kit (Nichirei, Tokyo, Japan) according to the manufacturer's instructions. The slide was then counterstained with hematoxylin. Lesions, in which all or most cells showed dark brownnuclear staining, were considered positive [26]. All samples were observed at a magnification of $400 \times$. Samples with $10 \%$ or higher staining for p53 in the nucleus were considered p53-positive.

\subsection{Statistical analyses}

Differences between diagnosis for 53 BP1-NF, LF, and 53 BP1-foci in Ki-67-positive nuclei were assessed by analysis of variance (ANOVA) and Tukey's multiple comparison tests. Receiver operating characteristic (ROC) analysis was performed for evaluating differences between LG-IN and HG-IN. Youden index was used to identify optimal cut-points on the ROC curve. Differences between clinicopathological parameters for $53 \mathrm{BP} 1$-foci, LF, and $53 \mathrm{BP} 1$-foci in Ki-67-positive nuclei were assessed either by two-tailed Student's $t$-test, ANOVA, or the coefficient of correlation. $P$-values $<0.05$ were considered statistically significant. Analyses were performed using the GLM, LOGISTIC, TTEST, and CORR tests in the SAS software package (version 9.4; SAS Institute, Inc., Cary, NC, USA) . 

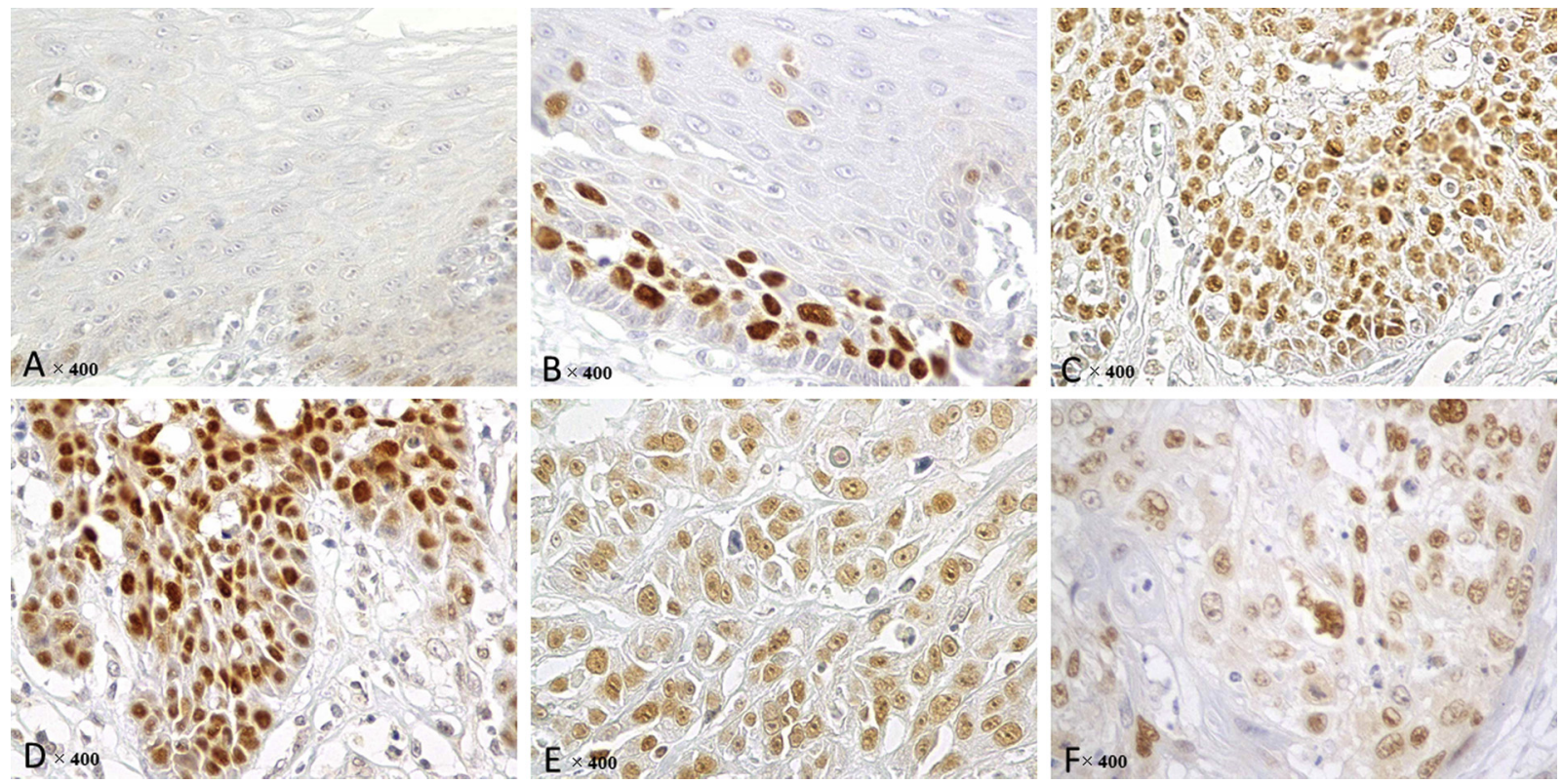

Fig. 1. Representative image of p53 immunohistochemistry. (a) Normal epithelium, (b) LG-IN, (c) HG-IN, (d) CIS, (e) surface, and (f) invasive front of SCC.

\section{Results}

\subsection{Expression of p53 and total 53 BP1 in esophageal lesions}

To confirm the accuracy of pathological diagnosis, p53 expression was first assessed in normal epithelium, LG-IN, HG-IN, and CIS samples (Fig. 1, Table 1). Normal epithelium did not exhibit p53 expression, whereas the basal layers of LG-IN were positive for p53 (11.1\%). HG-IN showed p53 staining from the basal layers to the surface of the epithelium (55.6\%), and a similar p53 expression pattern was observed in CIS (58.3\%), consistent with the enhanced expression of mutated p53 during carcinogenesis.

We then examined the difference in GIN between normal epithelium, LG-IN, HG-IN, CIS, and SCC using 53 BP1 as a marker for DDR (Fig. 3). Interestingly, the pathologically normal epithelium showed a modest expression of 53 BP1-NF (16.1\%), even though it was negative for p53 expression. Meanwhile, 53 BP1 expression in LG-IN (31.0\%) was significantly higher than in normal esophagus $(P=0.0107$; Table 2). In LG-IN, 53 BP1-NF expression was observed in the lower half of the epithelium, which was similar to the p53 expression pattern in the same area (Fig. 4). 53 BP1 expression levels increased as the tumor progressed; with normal squamous epithelium showing $16.1 \%$ expression of 53 BP1-NF, which increased to $31.0 \%$ in LG-IN, 34.5\% in HG-IN, $35.2 \%$ in CIS, and $41.7 \%$ on the surface lesion of invasive SCC $(P=0.0002$; Table 2). The $53 \mathrm{BP} 1-\mathrm{NF}$ expression rate was lower in the deep SCC lesions than on the surface of the tumor (Table 2), suggesting a lack of DNA damage repair in progressive states of SCC.

\subsection{Expression of 53 BP1 LF in esophageal lesions}

$53 \mathrm{BP} 1 \mathrm{LF}$ are larger than $1 \mu \mathrm{m}$ in diameter and represent sites of intensive GIN. Previous observations suggested that LF represents an extreme aggregation of $53 \mathrm{BP} 1$ at sites of DNA damage with incomplete repair [23]. Normal esophageal epithelium showed minimal expression of discrete 53 BP1 LF, whereas the number of LF increased with malignant progression of neoplasia (Table 2, Fig. 5). Histological staging of tumor progression from normal epithelium to SCC (surface) significantly correlated with the number of 53 BP1-LF $(P<0.0001$, Table 2). Furthermore, a statistically significant increase in 53 BP1-LF expression was observed between normal/LG-IN vs. HG-IN/CIS/SCC $(P=0.0107)$, suggesting increased GIN in HG-IN compared with LG-IN. In contrast, there was no statistically significant difference in 53 BP1-LF expression between HG-IN and CIS, or CIS and invasive SCC.
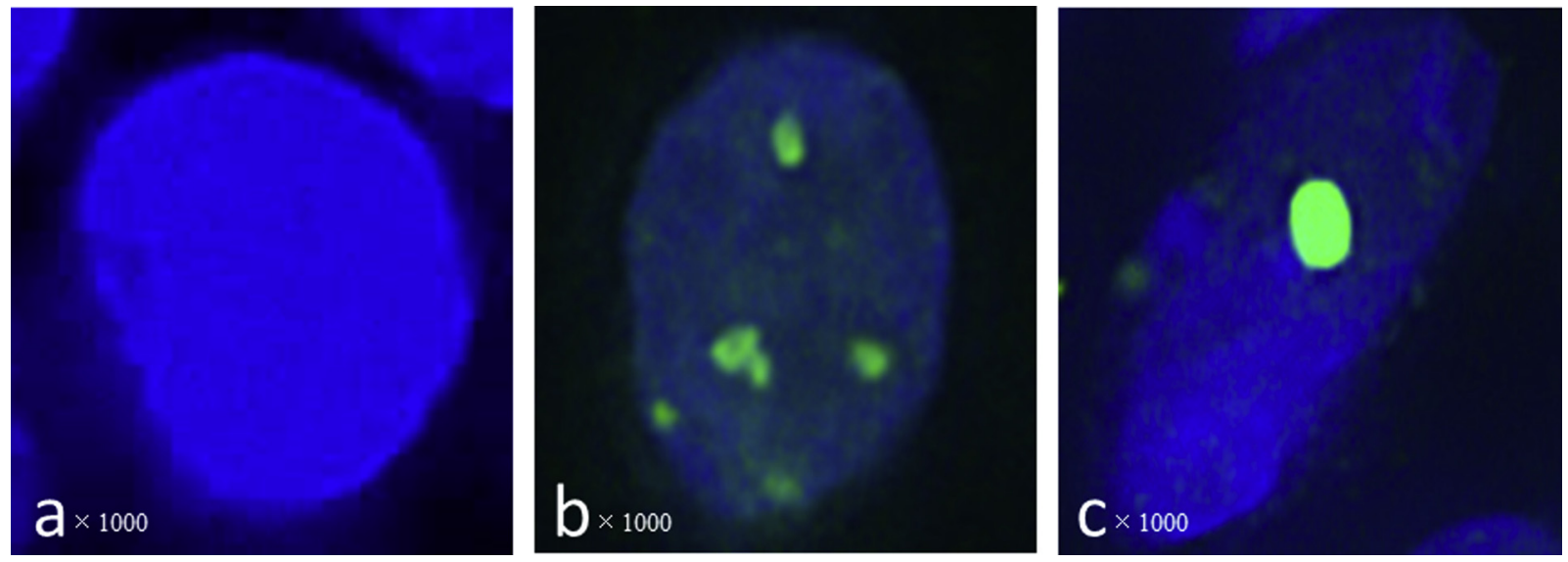

Fig. 2. Representative image of 53 BP1 nuclear expression. (a) Stable type: no expression or slightly diffuse; (b) unstable type : discrete 53 BP1-nuclear foci (NF); and (c) large focus (LF) type: discrete NF larger than $1.0 \mu \mathrm{m}$. 

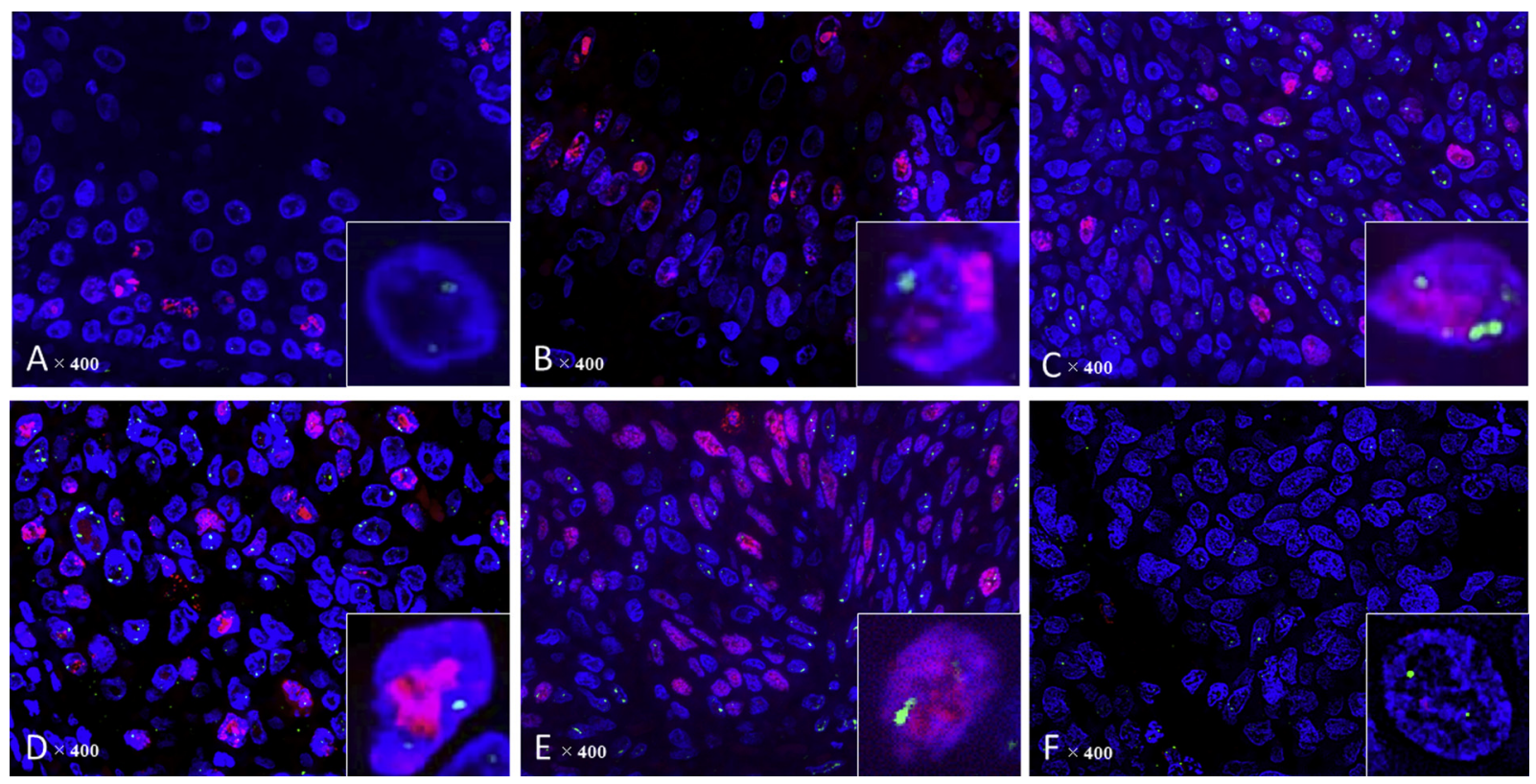

Fig. 3. Representative image of double-label immunofluorescence staining of 53 BP1 and Ki-67 in the esophageal epithelium. 53 BP1: green, Ki-67: red. (a) Normal epithelium, (b) LG-IN, (c) HG-IN, (d) CIN, and (e) surface and (f) invasive front of SCC.

\subsection{Expression of 53 BP1 in Ki-67-positive esophageal epithelium}

We next assessed the expression pattern of $53 \mathrm{BP} 1$ in combination with Ki-67 staining. Since DDR occurs during the G0 phase under physiological conditions, 53 BP1 expression during the growth phase (Ki-67-positive cells) suggests unregulated DDR [23]. We found increased 53 BP1-foci in Ki-67-positive nuclei during tumor progression from normal epithelium to SCC $(P=0.0019$; Table 2). These results suggest that not only the number of $53 \mathrm{BP} 1-\mathrm{NF}$ increase during epithelial carcinogenesis but that abnormally timed DDR increased as well.

\subsection{Distinguishing $L G-I N$ and $H G-I N$ using 53 BP1 as a marker}

Based on our observations, we tested the possibility of distinguishing LG-IN and HG-IN via-53 BP1 immunostaining. Total 53 BP1$\mathrm{NF}$ and 53 BP1-NF in Ki-67-positive nuclei rates were unable to distinguish between LG-IN and HG-IN, although both showed an increasing tendency during carcinogenesis (Table 2). On the other hand, our analysis revealed that when the cut off value was set to an LF expression rate of $7.762 \%$, the sensitivity and specificity of distinguishing between the two groups was 1.000 and 0.556 , respectively (Fig. 5). When the cut off value was set to an LF expression rate of $6.49 \%$, the sensitivity and specificity were 0.8889 and 0.6667 , respectively.

\subsection{Association between the type of $53 \mathrm{BP} 1 / \mathrm{Ki}-67$ expression and clinicopathological factors}

The association between the overall proportion of 53 BP1-NF, LF, and Ki-67-positive cells, as well as 53 BP1-NF in Ki-67-positive cells with the clinicopathological factors including patient age, tumor location, differentiation of the tumor, TNM stage, lymph node metastasis, distant metastasis, lymphatic invasion, and vascular invasion were analyzed. When all 61 lesions from 22 surgically resected cases were examined, the rate of LF was positively associated with age (Coefficient of correlation $=0.251, P=0.0488$ ), but not with other factors. When limited to SCC (surface), the rate of LF and 53 BP1-NF in Ki-67-positive cells tended to decrease as the tumor cells turned from well-differentiated to moderately- or poorly-differentiated carcinoma, although the differences were not statistically significant $(P=0.0764$ and $P=0.0967$, respectively, by ANOVA). Expression of 53 BP1 was not influenced by any of the TNM factors, although the number of Ki-67positive cells was significantly higher in the cases that presented with lymphatic invasion compared to those without lymphatic invasion $(P=0.0382$, by $t$-test $)$.

\section{Discussion}

The present study used 53 BP1 expression levels to demonstrate

Table 2

Focus pattern of 53 BP1 in the esophageal epithelium carcinogenesis process.

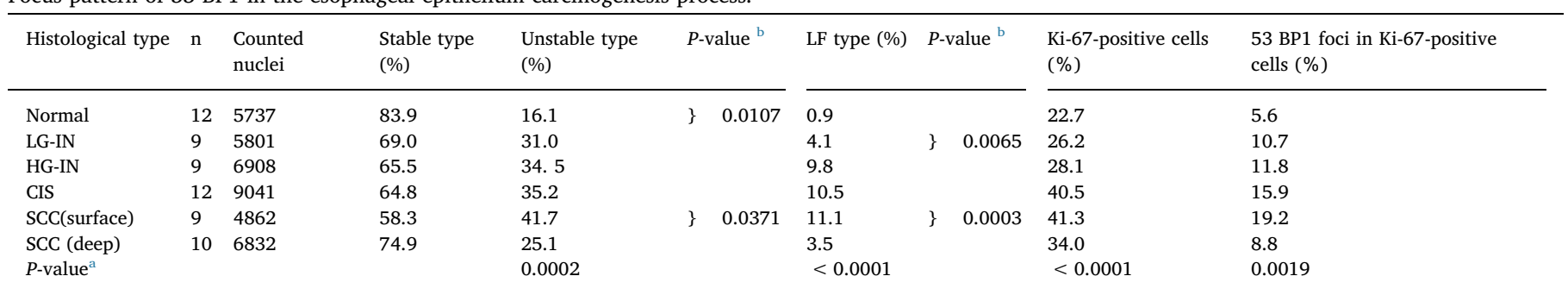

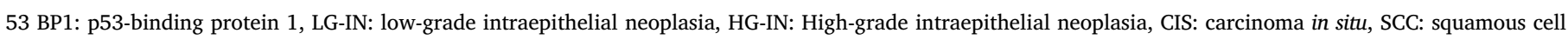
carcinoma, LF: large foci.

a Assessed by analysis of variance (ANOVA).

b Assessed by Tukey's multiple comparison test. 

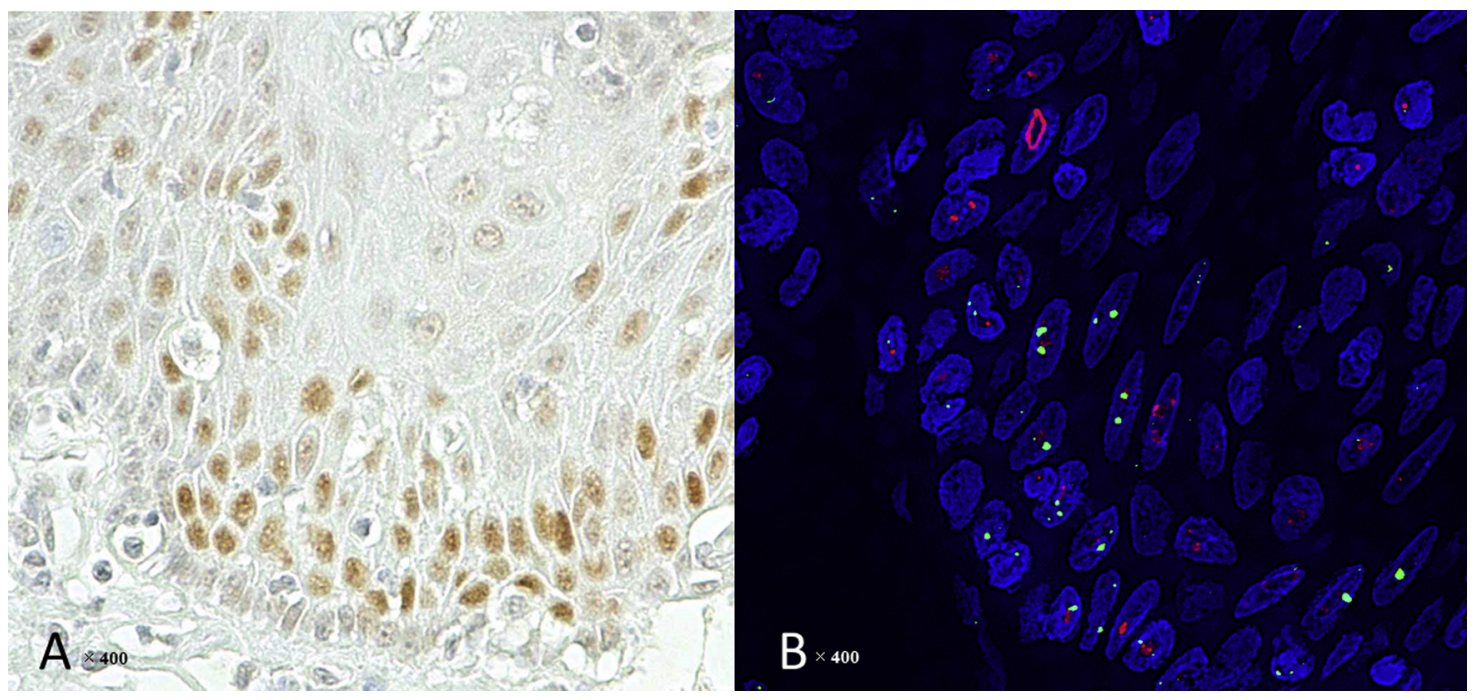

Fig. 4. Expression pattern of p53 and 53 BP1 in an LG-IN. (a) Immunohistochemistry of p53. (b) Double-label immunofluorescence staining of 53 BP1 (green) and Ki-67 expression (red).

distinct DNA damage patterns during esophageal carcinogenesis (Fig. 6). We found that the number of discrete 53 BP1-NF-a cytological marker of GIN [19]—in esophageal epithelium progressively increased as the tumor progressed from normal epithelium through LG-IN, HG-IN, CIS, and finally SCC. 53 BP1 is classified as an adaptor/mediator within the DDR mechanism, which is required for processing of the DDR signal and as a platform for the recruitment of other repair factors [27]. During physiological DNA repair, 53 BP1-NF disappear once the damage is fixed. However, the dysfunction of down-stream mediators of DNA repair induces $53 \mathrm{BP} 1$ accumulation at the DNA damage site. Thus the formation of $53 \mathrm{BP} 1-\mathrm{LF}$ is thought to indicate failure or prolonged inability to efficiently recover from DSB generation [23]. Our results are consistent with those of a recent study by Liu et al. [2], wherein WGS data and immunofluorescence were compared, and the number of genetic mutations in esophageal neoplasia was found to be positively associated with $\gamma \mathrm{H} 2 \mathrm{AX}$, which is a DDR marker.

In the current study, 53 BP1-NF were observed in $16 \%$ of the morphologically normal epithelium, although LF type of 53 BP1 was rarely detected. The esophagus is consistently exposed to stimulation from food, beverages, alcohol, and tobacco. 53 BP1-NF expression in the tumor-free area may represent physiological DDR in these circumstances, especially in patients who have esophageal SCC. Further studies are required to elucidate the differences in 53 BP1 expression in non-neoplastic lesions between healthy subjects and patients with SCC. It has been suggested that HG-IN, as well as CIS, should be subjected to endoscopic treatment [5]. Furthermore, according to the most recent Japanese guideline on esophageal carcinoma, HG-IN is histologically defined as a CIS [5]. Notably, our findings demonstrated that the number of discrete 53 BP1-NF in the esophageal epithelium was significantly different between LG-IN and HG-IN, but not between HG-IN and CIS. Thus, our findings support the abovementioned treatment approach as the DDR of HG-IN and CIS showed similar patterns that are distinct from LG-IN.

Ki-67, expressed during all cell cycle stages except G0, is a widely used marker of cell proliferation [28,29]. In the present study, the number of 53 BP1-NF in Ki-67-positive cells also increased as the tumor progressed from normal epithelium through LG-IN, HG-IN, CIS, indicating a decrease in physiological DDR and an increase in pathological DDR. These findings support previous observations regarding the accumulation of GIN during carcinogenesis [23,25]. Our study suggests

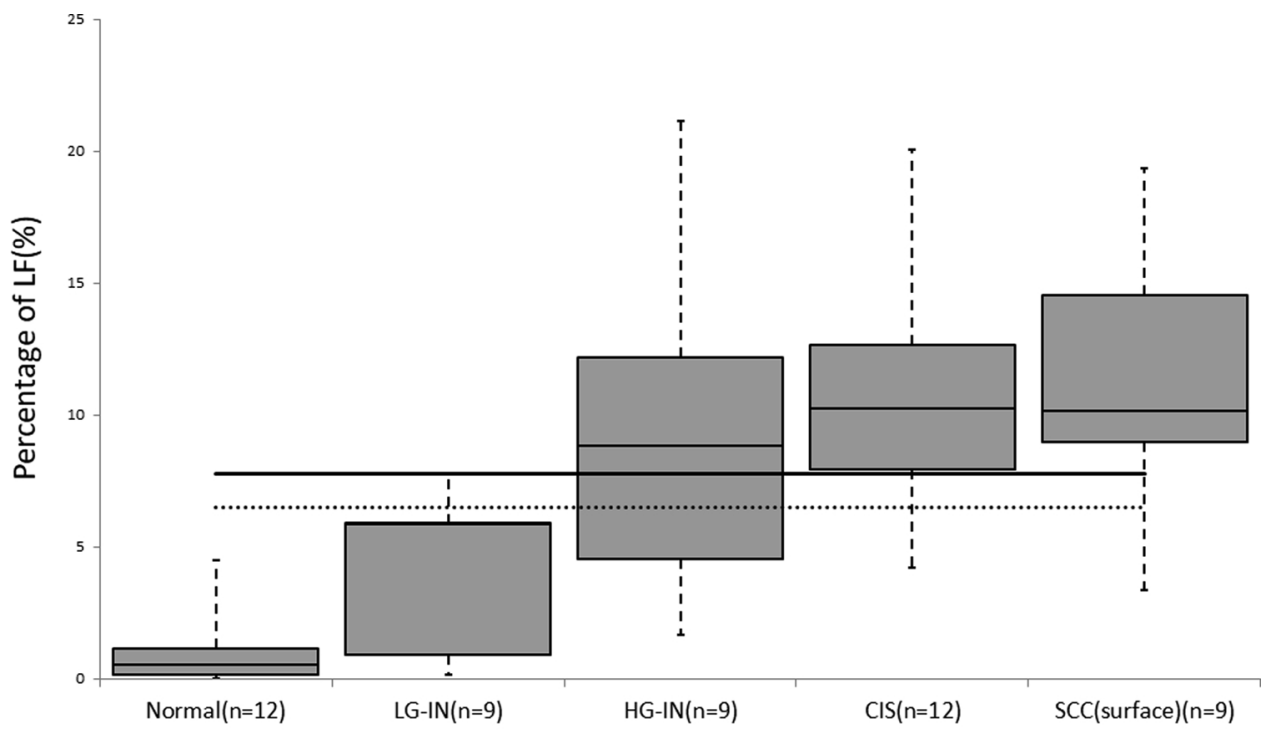

Fig. 5. The utility of 53 BP1-LF in distinguishing between LG-IN and HG-IN examined via Fisher's test. The solid line indicates the cut off LF expression rate of $7.762 \%$; sensitivity and specificity for distinguishing LG-IN $(\mathrm{n}=9)$ and HG-IN $(\mathrm{n}=9)$ were 1.000 and 0.556 , respectively. The dotted line indicates an LF expression rate of $6.49 \%$; sensitivity and specificity for distinguishing between LG-IN and HG-IN were 0.8889 and 0.6667 , respectively.

Histological type 


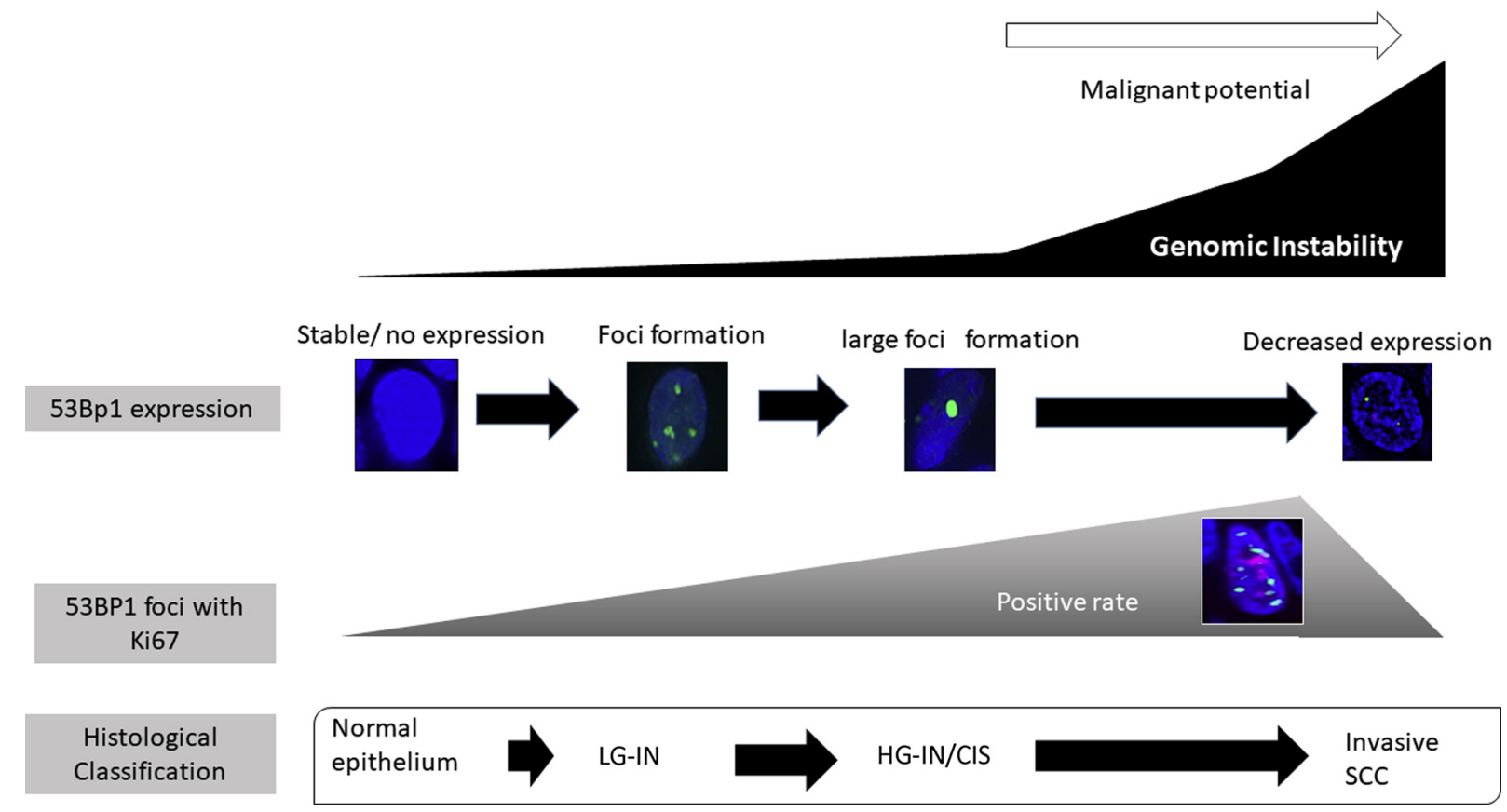

Fig. 6. Schematic representation of 53 BP1 expression in esophageal squamous cell neoplasms, and the proposed degree of genomic instability. 53 BP1-foci accumulate in LG-IN and continue to increase in number during progression to SCC, indicating increased stepwise genomic instability. Large foci, an indicator of intensive genomic instability, increase significantly in HG-IN/CIS. Once the tumor reaches an invasive state, the ability to respond to DDR is lost, leading to decreased 53 BP1 expression.

that DDR - represented by the accumulation of 53 BP1-NF - may have already occurred at the precancerous stage during esophageal carcinogenesis. In addition, increased GIN based on a disrupted DDR, indicated by the co-localization of 53 BP1 and Ki-67, may allow further accumulation of other genomic alterations, triggering progression to invasive cancer through the acceleration of cell growth/replication stress [3,23].

Furthermore, the proportion of 53 BP1-NF- and 53 BP1-NF in Ki-67positive cells tended to decrease in deep SCC lesions, which is in line with our previous observations on cervical malignancy [23]. The exact mechanism for this phenomenon requires further investigation, although studies have shown genetic and epigenetic intra-tumor heterogeneity in esophageal neoplasms [30,31]. The physiological DDR pathway in invasive tumors is impaired, enabling these tumors to escape DNA repair and apoptosis induction [32]. Therefore, we speculate that cells at the tumor front that exhibit invasive patterns lack the ability to respond to DNA damage, possibly indicating increased GIN and more aggressive phenotypes (Fig. 6).

Further studies are required to elucidate the molecular mechanism underlying the uncoupling of cell cycle progression in the event of increased DNA damage during advanced phases of carcinogenesis.

\section{Conclusions}

Our study demonstrated a stepwise increase in 53 BP1-NF during carcinogenesis. We also revealed the difference in GIN between LG-IN and HG-IN based on 53 BP1-LF expression. As 53 BP1-NF reflects DNA DSBs, we hypothesize that it represents the level of GIN in squamous cell neoplasms in the esophagus.

\section{Authors' contributions}

NU, SM, and TI performed the experiments; NU and YA drafted the manuscript; KM and HK provided critical input regarding the manuscript; HK performed the statistical analyses; MN conceptualized the study and conducted the experiments. All authors read and approved the final version of the manuscript prior to submission.

\section{Funding sources}

This work was supported in part by the Atomic Bomb Disease Institute of Nagasaki University, a Grant-in-Aid for Scientific Research from the Japanese Ministry of Education, Science, Sports, and Culture (Grant no. 23790406) and the Program of the Network-Type Joint Usage/Research Center for Radiation Disaster Medical Science.

\section{Declaration of Competing Interest}

The authors have no competing interests.

\section{Acknowledgments}

We wish to thank Editage (www.editage.jp) for English language editing.

\section{Appendix A. Supplementary data}

Supplementary material related to this article can be found, in the online version, at doi:https://doi.org/10.1016/j.prp.2019.152601.

\section{References}

[1] E.C. Smyth, J. Lagergren, R.C. Fitzgerald, F. Lordick, M.A. Shah, P. Lagergren, D. Cunningham, Oesophageal cancer, Nat. Rev. Dis. Prim. 3 (2017) 17048, https:// doi.org/10.1038/nrdp.2017.48.

[2] F.T. Bosman, World Health Organization., International Agency for Research on Cancer., WHO Classification of Tumours of the Digestive System, International Agency for Research on Cancer, 2010, http://publications.iarc.fr/Book-And-ReportSeries/Who-Iarc-Classification-Of-Tumours/WHO-Classification-Of-Tumours-OfThe-Digestive-System-2010.

[3] X. Liu, M. Zhang, S. Ying, C. Zhang, R. Lin, J. Zheng, G. Zhang, D. Tian, Y. Guo, C. Du, Y. Chen, S. Chen, X. Su, J. Ji, W. Deng, X. Li, S. Qiu, R. Yan, Z. Xu, Y. Wang, Y. Guo, J. Cui, S. Zhuang, H. Yu, Q. Zheng, M. Marom, S. Sheng, G. Zhang, S. Hu, R. Li, M. Su, Genetic alterations in esophageal tissues from squamous dysplasia to carcinoma, Gastroenterology. 153 (2017) 166-177, https://doi.org/10.1053/j. gastro.2017.03.033.

[4] Y. Shimizu, M. Takahashi, T. Yoshida, S. Ono, K. Mabe, M. Kato, M. Asaka, N. Sakamoto, Endoscopic resection (endoscopic mucosal resection/ endoscopic 
submucosal dissection) for superficial esophageal squamous cell carcinoma: current status of various techniques, Dig. Endosc. 25 (2013) 13-19, https://doi.org/10. 1111/j.1443-1661.2012.01408.x.

[5] Y. Tanaka, K. Yoshida, T. Suetsugu, T. Imai, N. Matsuhashi, K. Yamaguchi, Recent advancements in esophageal cancer treatment in Japan, Ann. Gastroenterol. Surg. 2 (2018) 253-265, https://doi.org/10.1002/ags3.12174.

[6] P. Bork, K. Hofmann, P. Bucher, A.F. Neuwald, S.F. Altschul, E.V. Koonin, A superfamily of conserved domains in DNA damage-responsive cell cycle checkpoint proteins, FASEB J. 11 (1997) 68-76 (accessed March 18, 2019), http://www.ncbi. nlm.nih.gov/pubmed/9034168.

[7] W.S. Joo, P.D. Jeffrey, S.B. Cantor, M.S. Finnin, D.M. Livingston, N.P. Pavletich, Structure of the $53 \mathrm{BP} 1 \mathrm{BRCT}$ region bound to $\mathrm{p} 53$ and its comparison to the Brca1 BRCT structure, Genes Dev. 16 (2002) 583-593, https://doi.org/10.1101/gad. 959202.

[8] L.B. Schultz, N.H. Chehab, A. Malikzay, T.D. Halazonetis, p53 binding protein 1 (53 BP1) is an early participant in the cellular response to DNA double-strand breaks, J. Cell Biol. 151 (2000) 1381-1390 (accessed March 18, 2019), http://www.ncbi.nlm. nih.gov/pubmed/11134068.

[9] M.T. Perfetti, B.M. Baughman, B.M. Dickson, Y. Mu, G. Cui, P. Mader, A. Dong, J.L. Norris, S.B. Rothbart, B.D. Strahl, P.J. Brown, W.P. Janzen, C.H. Arrowsmith, G. Mer, K.M. McBride, L.I. James, S.V. Frye, Identification of a fragment-like small molecule ligand for the methyl-lysine binding protein, 53 BP1, ACS Chem. Biol. 10 (2015) 1072-1081, https://doi.org/10.1021/cb500956g.

[10] I.M. Ward, K. Minn, K.G. Jorda, J. Chen, Accumulation of checkpoint protein 53 BP1 at DNA breaks involves its binding to phosphorylated histone H2AX, J. Biol Chem. 278 (2003) 19579-19582, https://doi.org/10.1074/jbc.C300117200.

[11] I. Rappold, K. Iwabuchi, T. Date, J. Chen, Tumor suppressor p53 binding protein 1 (53 BP1) is involved in DNA damage-signaling pathways, J. Cell Biol. 153 (2001) 613-620 (accessed March 18, 2019), http://www.ncbi.nlm.nih.gov/pubmed/ 11331310.

[12] L. Anderson, C. Henderson, Y. Adachi, Phosphorylation and rapid relocalization of 53 BP1 to nuclear foci upon DNA damage, Mol. Cell. Biol. 21 (2001) 1719-1729, https://doi.org/10.1128/MCB.21.5.1719-1729.2001.

[13] Z. Xia, J.C. Morales, W.G. Dunphy, P.B. Carpenter, Negative cell cycle regulation and DNA damage-inducible phosphorylation of the BRCT protein 53 BP1, J. Biol. Chem. 276 (2001) 2708-2718, https://doi.org/10.1074/jbc.M007665200.

[14] Y. Shiloh, M.B. Kastan, ATM: genome stability, neuronal development, and cancer cross paths, Adv. Cancer Res. 83 (2001) 209-254 (accessed March 18, 2019), http://www.ncbi.nlm.nih.gov/pubmed/11665719.

[15] Y. Xu, D. Baltimore, Dual roles of ATM in the cellular response to radiation and in cell growth control, Genes Dev. 10 (1996) 2401-2410 (accessed March 18, 2019), http://www.ncbi.nlm.nih.gov/pubmed/8843193.

[16] Y. Xu, E.M. Yang, J. Brugarolas, T. Jacks, D. Baltimore, Involvement of p53 and p21 in cellular defects and tumorigenesis in Atm-/- mice, Mol. Cell. Biol. 18 (1998) 4385-4390 (accessed March 18, 2019), http://www.ncbi.nlm.nih.gov/pubmed/ 9632822.

[17] T.A. Mochan, M. Venere, R.A. DiTullio, T.D. Halazonetis, 53 BP1 and NFBD1/ MDC1-Nbs1 function in parallel interacting pathways activating ataxia-telangiectasia mutated (ATM) in response to DNA damage, Cancer Res. 63 (2003) 8586-8591 (accessed March 18, 2019), http://www.ncbi.nlm.nih.gov/pubmed/ 14695167.

[18] H.A. Rakhorst, W.M.W. Tra, S.T. Posthumus-Van Sluijs, S.E.R. Hovius, P.C. Levendag, R. Kanaar, S.O.P. Hofer, Quantitative analysis of radiation-induced DNA break repair in a cultured oral mucosal model, Tissue Eng. 12 (2006) 3395-3403, https://doi.org/10.1089/ten.2006.12.3395.

[19] K. Suzuki, S. Yokoyama, S. Waseda, S. Kodama, M. Watanabe, Delayed reactivation of p53 in the progeny of cells surviving ionizing radiation, Cancer Res. 63 (2003)
936-941 (accessed March 18, 2019), http://www.ncbi.nlm.nih.gov/pubmed/ 12615706

[20] Y. Naruke, M. Nakashima, K. Suzuki, H. Kondo, T. Hayashi, M. Soda, I. Sekine, Genomic instability in the epidermis induced by atomic bomb (A-bomb) radiation, Cancer. 115 (2009) 3782-3790, https://doi.org/10.1002/cncr.24405.

[21] M. Nakashima, K. Suzuki, S. Meirmanov, Y. Naruke, M. Matsuu-Matsuyama, K. Shichijo, V. Saenko, H. Kondo, T. Hayashi, M. Ito, S. Yamashita, I. Sekine, Foci formation of P53-binding protein 1 in thyroid tumors: activation of genomic instability during thyroid carcinogenesis, Int. J. Cancer 122 (2007) 1082-1088, https://doi.org/10.1002/ijc.23223.

[22] Y. Naruke, M. Nakashima, K. Suzuki, M. Matsuu-Matsuyama, K. Shichijo, H. Kondo I. Sekine, Alteration of p53-binding protein 1 expression during skin carcinogenesis: association with genomic instability, Cancer Sci. 99 (2008) 946-951, https://doi. org/10.1111/j.1349-7006.2008.00786.x.

[23] K. Matsuda, S. Miura, T. Kurashige, K. Suzuki, H. Kondo, M. Ihara, H. Nakajima, H. Masuzaki, M. Nakashima, Significance of p53-binding protein 1 nuclear foci in uterine cervical lesions: endogenous DNA double strand breaks and genomic instability during carcinogenesis, Histopathology. 59 (2011) 441-451, https://doi. org/10.1111/j.1365-2559.2011.03963.x.

[24] Z. Mussazhanova, Y. Akazawa, K. Matsuda, K. Shichijo, S. Miura, R. Otsubo, M. Oikawa, K. Yoshiura, N. Mitsutake, T. Rogounovitch, V. Saenko, Z. Kozykenova, B. Zhetpisbaev, D. Shabdarbaeva, N. Sayakenov, B. Amantayev, H. Kondo, M. Ito, M. Nakashima, Association between p53-binding protein 1 expression and genomic instability in oncocytic follicular adenoma of the thyroid, Endocr. J. 63 (2016) 457-467, https://doi.org/10.1507/endocrj.EJ15-0629.

[25] Z. Mussazhanova, K. Matsuda, Y. Naruke, N. Mitsutake, B. Stanojevic, T. Rougounovitch, V. Saenko, K. Suzuki, E. Nishihara, M. Hirokawa, M. Ito, M. Nakashima, Significance of p53-binding protein 1 (53 BP1) expression in thyroid papillary microcarcinoma: association with $B R A F^{V}{ }^{600 E}$ mutation status, Histopathology. 63 (2013), https://doi.org/10.1111/his.12233 n/a-n/a.

[26] M. Yasuda, H. Kuwano, M. Watanabe, Y. Toh, S. Ohno, K. Sugimachi, p53 expression in squamous dysplasia associated with carcinoma of the oesophagus: evidence for field carcinogenesis, Br. J. Cancer 83 (2000) 1033-1038, https://doi.org/10. 1054/bjoc. 2000.1443.

[27] A. Gupta, C.R. Hunt, S. Chakraborty, R.K. Pandita, J. Yordy, D.B. Ramnarain, N. Horikoshi, T.K. Pandita, Role of 53 BP1 in the regulation of DNA double-strand break repair pathway choice, Radiat. Res. 181 (2014) 1-8, https://doi.org/10. 1667/RR13572.1.

[28] S. Wayne, R.A. Robinson, Upper aerodigestive tract squamous dysplasia: correlation with p16, p53, pRb, and Ki-67 expression, Arch. Pathol. Lab. Med. 130 (2006) 1309-1314, https://doi.org/10.1043/1543-2165(2006)130[1309:UATSDC]2.0. $\mathrm{CO} ; 2$.

[29] S. Mithani, W. Mydlarz, F. Grumbine, I. Smith, J. Califano, Molecular genetics of premalignant oral lesions, Oral Dis. 13 (2007) 126-133, https://doi.org/10.1111/j 1601-0825.2006.01349.x.

[30] D.-C. Lin, J.-J. Hao, Y. Nagata, L. Xu, L. Shang, X. Meng, Y. Sato, Y. Okuno, A.M. Varela, L.-W. Ding, M. Garg, L.-Z. Liu, H. Yang, D. Yin, Z.-Z. Shi, Y.-Y. Jiang, W.-Y. Gu, T. Gong, Y. Zhang, X. Xu, O. Kalid, S. Shacham, S. Ogawa, M.-R. Wang, H.P. Koeffler, Genomic and molecular characterization of esophageal squamous cell carcinoma, Nat. Genet. 46 (2014) 467-473, https://doi.org/10.1038/ng.2935.

[31] D.-C. Lin, M.-R. Wang, H.P. Koeffler, Genomic and epigenomic aberrations in esophageal squamous cell carcinoma and implications for patients, Gastroenterology. 154 (2018) 374-389, https://doi.org/10.1053/j.gastro.2017.06.066.

[32] J. Bartkova, F. Tort, K. Zieger, P. Guldberg, M. Sehested, Z. Hor, Z. Horejsí, K. Koed, A. Krämer, J.M. Nesland, C. Lukas, T. Ørntoft, J. Lukas, J. Bartek, DNA damage response as a candidate anti-cancer barrier in early human tumorigenesis, Nature. (2005), https://doi.org/10.1038/nature03482. 